\title{
Editorial
}

\section{Advanced Signal Processing for Digital Subscriber Lines}

\author{
Raphael Cendrillon, ${ }^{1}$ lain Collings, ${ }^{2}$ Tomas Nordström, ${ }^{3}$ Frank Sjöberg, ${ }^{4}$ Michail Tsatsanis, ${ }^{5}$ and Wei Yu ${ }^{6}$ \\ ${ }^{1}$ Marvell Hong Kong Ltd., Hong Kong \\ ${ }^{2}$ CSIRO Information Communication Technologies Center, Australia \\ ${ }^{3}$ Telecommunications Research Center Vienna (ftw.), Donau-City-StraBe 1, 1220 Vienna, Austria \\ ${ }^{4}$ Division of Signal Processing, Luleå University of Technology, and Upzide Labs, Luleå, Sweden \\ ${ }^{5}$ Aktino Inc., Irvine, California, USA \\ ${ }^{6}$ Electrical and Computer Engineering Department, University of Toronto, 10 King's College Road, Toronto, ON Canada, M5S $3 G 4$
}

Received 27 January 2006; Accepted 27 January 2006

Copyright (c) 2006 Raphael Cendrillon et al. This is an open access article distributed under the Creative Commons Attribution License, which permits unrestricted use, distribution, and reproduction in any medium, provided the original work is properly cited.

The recent deployment of digital subscriber line (DSL) technology around the world is rapidly making broadband access for the mass consumer market a reality. The ever-growing customer demand for higher data rates has been fueled by the popularity of applications like peer-to-peer (P2P) filesharing networks and video-streaming and high-definition television (HDTV). DSL technology allows telephone operators to get maximum leverage out of their existing infrastructure by delivering broadband access over existing twistedpair telephone lines. At the heart of DSL lies a plethora of signal processing techniques which enable such high-speed transmission to be achieved over a medium originally designed with only voice-band transmission in mind. These advanced signal processing techniques address many challenges that exist in DSL networks today, such as the near-end and far-end crosstalk (NEXT/FEXT), impulse noise, peak-toaverage-power ratio (PAR), intersymbol and intercarrier interference (ISI/ICI), radio-frequency interference (RFI), and so forth. The goal of this special issue is to discuss the stateof-the-art and recent advances in signal processing techniques for DSL.

The special issue consists of fifteen papers on a range of topics. The first set of papers focuses on the area of dynamic spectrum management (DSM). In a conventional DSL deployment, the transmit spectrum for all modems in a bundle are fixed to a predetermined level. As DSL deployment becomes increasingly heterogeneous, crosstalk produced by modems under a fixed spectrum can be a source of significant interference. Dynamic spectrum management aims to improve the data rates and reaches of conventional DSL systems by adaptively varying transmit power-spectral density according to geographic locations and the crosstalk channel characteristics of the subscribers in each bundle. This issue contains six papers on DSM. In "The worst-case interference in DSL systems employing dynamic spectral management," Brady and Cioffi answer the question of what the worst-case crosstalk interference is for a given DSL line. They characterize the performance of the system under the worstcase noise using a game theory technique. In "Joint multiuser detection and optimal spectrum balancing for digital subscriber lines," Chan and Yu study the optimal spectrum management technique for a scenario in which crosstalk may also be partially cancelled using advanced crosstalk cancellation techniques. In the next three papers, practical spectrum management techniques are investigated. In "Spectrally compatible iterative water-filling," Verlinden et al. study a system in which spectral allocation scheme is constrained by additional spectrum compatibility requirements and propose a new scheme based on an earlier algorithm called iterative water-filling. In "The normalizedrate iterative algorithm: a practical dynamic spectrum management method for DSL," Statovci et al. propose a new low-complexity technique for spectrum balancing and frequency partition in a DSL bundle. In "ADSL transceivers applying DSM and their nonstationary noise robustness," Van den Bogaert et al. report the performance of practical transceivers implementing dynamic spectrum management and study their robustness against nonstationary noise. Finally, from a theoretical perspective, the paper "Analysis of iterative water-filling algorithm for multiuser power control 
in digital subscriber lines," by Luo and Pang, takes a new look at the iterative water-filling algorithm and gives a novel interpretation of the algorithm based on optimization theory.

The next paper in the special issue deals with crosstalk cancellation. In a DSL deployment, when coordination among the transmit- or receive-modems is possible, further data improvement may be obtained via crosstalk cancellation. In the paper "Alien crosstalk cancellation for multipair digital subscriber line systems," Ginis and Peng give an overview of this area and propose a new crosstalk cancellation technique that takes advantage of the noise correlation among the multiple receivers.

The practical success of dynamic spectrum management and crosstalk cancellation depends very much on how accurately crosstalk channels may be modeled and identified in practice. Two papers of this special issue address this area. In "Crosstalk models for short VDSL2 lines from measured $30 \mathrm{MHz}$ data," Karipidis et al. propose measurement-based crosstalk models for VDSL. In "Error sign feedback as an alternative to pilots for the tracking of FEXT transfer functions in downstream VDSL," Louveaux and Van der Veen propose new ways of identifying the crosstalk channel using a novel feedback scheme.

Equalization and coding continue to be important research topics in DSL. In the area of time-domain equalization (TEQ), the paper "Iterative refinement methods for time-domain equalizer design" by Arslan et al. proposes a new method to reduce the implementation complexity of the TEQ. In the area of error-correcting coding for the DSL system, the paper "Near capacity coding for discrete multitone (DMT) systems with impulse noise" by Ardakani et al. proposes a methodology for the design of the newly emerged low-density parity-check (LDPC) codes for a DMT system, while addressing the practical DSL deployment issue of impulse noise. In "Fine-granularity loading schemes using adaptive Reed-Solomon coding for XDSLDMT systems," Panigrahi and Le-Ngoc propose a joint design of bit-loading and error-correcting code, and characterize the performance gain made possible by fractional bitloading.

The final set of three papers in this special issue deals with the area of modulation and transmitter design. The design of transmit window to minimize egress is studied in the paper by Cuypers et al. "Intra-symbol windowing for egress reduction in DMT transmitters." The peak-toaverage-power ratio is another important transmitter design issue for DMT systems. This is taken up in the paper "Designing tone reservation PAR reduction" by Andgart et al. DMT is not the only possible multicarrier modulation scheme for DSL. An alternative is proposed and studied in the paper "Cosine modulated multitone for very high-speed digital subscriber lines" by Lin and FarhangBoroujeny.

The continued growth of digital subscriber line technology worldwide is in part fueled by rapid advances in signal processing techniques. We hope that the readers will enjoy the collection of papers on this timely topic.
Finally, we wish to take this opportunity to acknowledge and to thank all anonymous reviewers, without whom the success of this special issue would not have been possible.

\section{Raphael Cendrillon Iain Collings \\ Tomas Nordström \\ Frank Sjöberg \\ Michail Tsatsanis \\ Wei Yu}

Raphael Cendrillon was born in Melbourne, Australia, in 1978. He received an Electrical Engineering degree (honours first class) from the University of Queensland, Australia, in 1999, and a Ph.D. in electrical engineering at the Katholieke Universiteit Leuven, Belgium, in 2004. His Ph.D. was awarded Summa Cum Laude with congratulations of the jury, an honor given to the top $5 \%$ of Ph.D. graduates. His research focuses on the application of multiuser communication theory to xDSL. In 2002, he was a Visiting Scholar at the Information Systems Laboratory, Stanford University, with Prof. John Cioffi. In 2005, Dr. Cendrillon was a postdoctoral Research Fellow at the University of Queensland, Australia. During this period, he was also a Visiting Research Fellow at Princeton University with Prof. Mung Chiang. He is now a senior DSP engineer with Marvell Hong Kong Ltd. He was awarded the Alcatel Bell Scientific Prize in 2004; IEEE Travel Grants in 2003, 2004, and 2005; the K.U. Leuven Bursary for Advanced Foreign Scholars in 2004; and the UniQuest Trailblazer Prize for Commercialization in 2005.

Iain Collings was born in Melbourne, Australia, in 1970. He received the B.E. degree in electrical and electronic engineering from the University of Melbourne, in 1992, and the Ph.D. degree in systems engineering from the Australian National University, in 1995. Currently he is a Science Leader in the CSIRO Information Communication Technologies Centre, Australia. Prior to this he was an Associate Professor at the Univer-

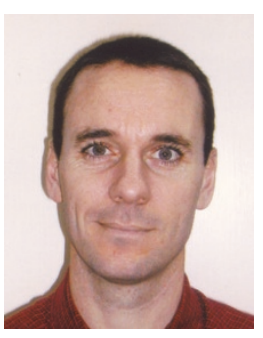
sity of Sydney (1999-2005); a Lecturer at the University of Melbourne (1996-1999); and a Research Fellow in the Australian Cooperative Research Centre for Sensor Signal and Information Processing (1995). His current research interests include mobile digital communications and broadband digital subscriber line communications; more specifically, synchronization, channel estimation, equalization, and multicarrier modulation, for time-varying and frequency-selective channels. He currently serves as an Editor for the IEEE Transactions on Wireless Communications, and as a Guest Editor for the EURASIP Journal on Advanced Signal Processing. He has also served as the Vice Chair of the Technical Program Committee for IEEE Vehicular Technology Conf. (Spring) 2006, as well as serving on a number of other TPCs and organizing committees of international conferences. He is also a founding organizer of the Australian Communication Theory Workshops 2000-2006. 
Tomas Nordström was born in Härnösand, Sweden, in 1963. He received the M.S.E.E. degree in 1988, the Licentiate degree in 1991, and the Ph.D. degree in 1995, all from Luleå University of Technology, Sweden. Currently, he is a key researcher and project manager at the Telecommunications Research Center Vienna (ftw). During 1995 and 1996, he was an Assistant Professor at Luleå University of Technology research-

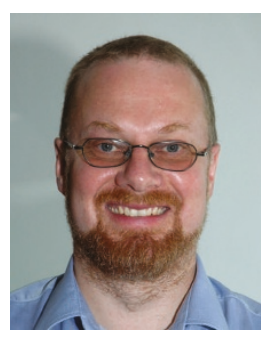
ing computer architectures, neural networks, and signal processing. Between 1996 and 1999, he was with Telia Research (the research branch of the Swedish incumbent telephone operator), where he developed broadband Internet communication over twisted copper pairs. He was instrumental in the development of the Zipper-VDSL concept (contributed to the standardization of VDSL in ETSI, ANSI, and ITU) and in the design of the Zipper-VDSL prototype modems. In December 1999, he joined $\mathrm{ftw}$, where he is the project Manager of the "Broadband wireline access" group. At ftw he has worked with various aspects of wireline communications like simulation of xDSL systems, cable measurements, RFI suppression, and exploiting the commonmode signal in xDSL. Currently his research is focused on "active copper resource management" which includes researching optimized usage of cable bundles and dynamic spectrum management.

Frank Sjöberg was born in Ume, Sweden, in 1970. He received the M.S. degree in computer science and the Ph.D. degree in signal processing from Luleå University of Technology, Luleå, Sweden, in 1995 and 2000, respectively. Between 2000 and 2002, he was with Telia Research AB, Luleå, Sweden. He currently works as a Researcher at Upzide Labs, and holds a position as an Assistant Professor in the Division of Signal Process-

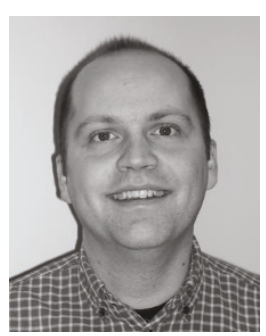
ing, Luleå University of Technology. His primary research interests are statistical signal processing and digital communication, with emphasis on wireline systems.

Michail Tsatsanis is a founder and Chief Scientist of Aktino, a company developing next generation DSL transceiver technology. Prior to that he was with Voyan Technology, where he served as Chief Scientist and Chief Technical Officer. From 1995 to 2000, he was with Stevens Institute of Technology, NJ, were he served as an Associate Professor in Electrical Engineering. He is the author of more than 80 peer reviewed

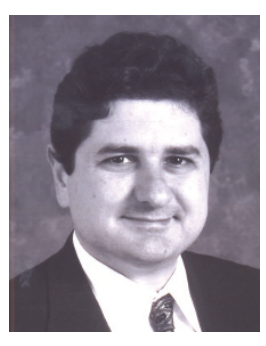
papers, three book chapters, and several patents. At Aktino he is leading a technology team that was first to successfully implement and produce a MIMO vectored transceiver in the DSL space. He has received a number of distinctions including the National Science Foundation CAREER Award and two IEEE Best Paper Awards. He has served the IEEE in various capacities including the position of Associate Editor for two IEEE Transactions and Chair of workshop organizing committees. He holds M.S. and Ph.D. degrees in electrical engineering from the University of Virginia.
Wei Yu received the B.A.S. degree in computer engineering and mathematics from the University of Waterloo, Waterloo, ON, Canada, in 1997, and M.S. and Ph.D. degrees in electrical engineering from Stanford University, Stanford, Calif, USA, in 1998 and 2002, respectively. Since 2002, he has been an Assistant Professor with the Electrical and Computer Engineering Department at the University of Toronto,

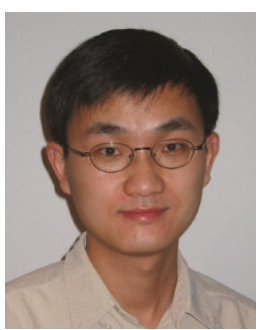
Toronto, ON, Canada, where he also holds a Canada Research Chair. His main research interests include multiuser information theory, coding, optimization, wireline communications, and wireless broadband access networks. He is currently an Associate Editor for the IEEE Transactions on Wireless Communications. 